\title{
Genomic features of oil model compounds biodegradation by Rhodococcus qingshengii strain VKM Ac-2784D
}

\author{
Petrushin I.S. ${ }^{1,2 *}$, Markova Y.A. ${ }^{1}$, Karepova M.S. ${ }^{1}$, Kudryavtseva Y.A. ${ }^{2}$, \\ Belovezhets L.A. ${ }^{3}$ \\ ${ }^{1}$ Siberian Institute of Plant Physiology and Biochemistry, Siberian Branch of the Russian Academy \\ of Sciences, Irkutsk, Russia \\ ${ }^{2}$ Irkutsk State University, Irkutsk, Russia \\ ${ }^{3}$ A.E. Favorsky Irkutsk Institute of Chemistry, Siberian Branch of the Russian Academy of Sciences, \\ Irkutsk, Russia \\ * email: ivan.kiel@gmail.com
}

Products of petroleum origin (oil and its components) are dangerous pollutants of the soil. Bacterial species of genus Rhodococcus have the ability to degrade a variety of hydrocarbons in contaminated soil and are useful for the bioremediation of polluted environments. We isolated the Rhodococcus qingshengii strain VKM Ac-2784D from the rhizosphere of couch grass (Elytrigia repens) which grows on oil-polluted site near Zalary, Irkutsk State, Russia (Belovezhets, 2017, 2020). This strain can effectively degrade oil and some model compounds (naphthalene, anthracene and phenanthrene). Such activity lowers the negative effect of soil pollution and recovers grow process of plants (Tretyakova, 2019). Although Rhodococcus spp. are well-known biosurfactant producers in temperate environments, the ability to synthesise surface-active agents is recognized as a general feature among cold-adapted microorganisms from this phylotype. We suggest that bioremediation activity of the strain in this study is associated with biosurfactants and phytohormones production.

This whole-genome shotgun sequence has been deposited at DDBJ/ENA/GenBank under the accession No. CP064920. We performed the search for biosynthetic gene clusters (BGCs) encoding secondary metabolites with web version 6.0 of antiSMASH and found 19 functional clusters (regions) on the chromosome. These gene clusters for heterobactin, ectoine, erythrochelin production, bacteriocin, ectoine, heterobactin A/heterobactin S2 (non-ribosomal peptide synthetase, NRPS). Ectoine may have a protectiverole, it helps bacteria to survive in extreme conditions (such as low temperatures and oil pollution).

The genomic features study of the Rhodococcus qingshengii strain VKM Ac-2784D helps to understand its hydrocarbons degrading mechanisms and broaden our knowledge about plant- bacteria interaction. 